Convergences francophones 6.2 (2020): 44-54

http://mrujs.mtroyal.ca/index.php/cf/index

\title{
Le fil des kilomètres (2013) de Christian Guay-Poliquin : la déconstruction du sens
}

\author{
Steven Urquhart \\ University of Lethbridge
}

Publié en 2013, le premier livre de l'auteur québécois Christian GuayPoliquin a été bien accueilli par la critique de la belle-province qui l'a comparé à L'Odyssée d'Homère et à Volkswagen Blues de Jacques Poulin. ${ }^{1}$ Histoire d'un narrateur anonyme qui traverse le continent d'ouest en est en voiture, suite à une panne d'électricité inexpliquée, pour revoir son père à la mémoire défaillante, $L e$ fil des kilomètres constitue effectivement une sorte de road novel à la fois épique et fascinant. ${ }^{2}$ Narré à la première personne, le roman s'organise en fonction du voyage transcontinental du protagoniste, un mécanicien, et puis d'un fantasme mythologique, dans lequel un jeune « mercenaire » anonyme se fait poursuivre par un minotaure dans un labyrinthe, tel Thésée. Présenté en arrière-plan du roman à travers neuf courts chapitres, ce fantasme semble renvoyer au rapport entre le protagoniste et son père, à son passé, et préfigurer la nature traumatisante du voyage qu'il entreprend. Destiné à réconcilier le narrateur avec son père, ce voyage finit par échouer in extremis et replonge le protagoniste dans un cauchemar familial et cyclique. Parti à la recherche d'une certaine plénitude suite au vide existentiel que provoque la panne, le narrateur retrouve enfin son père au bout de son chemin, mais n'arrive pas à le sauver.

Fort ironique, le voyage, qui commence déjà dans un cadre dystopique, aboutit, mais de façon tragique, et déconstruit ainsi la quête du Sens, la notion de progrès et l'idée qu'il est possible de revenir sur ses pas et de corriger le passé. Ayant pour point de départ un cul-de-sac, la route s'avère sans issue et remet en question la réalité du rêve américain où le fait d'aller de l'avant est synonyme de succès et de prospérité. En effet, le roman déconstruit le projet expansionniste occidental moderne remontant au Siècle des lumières; ${ }^{3}$ il expose les angles morts de la poussée en avant et laisse entendre que la machine socio-économique actuelle, nourrie par le pétrole, est cassée, voire ne fonctionne plus et que sa "réparation" ne se fera pas en revenant en arrière. Pris dans une sorte de cercle vicieux, l'individu et la collectivité doivent faire face à la nature aléatoire et imprévisible du monde et au mythe selon lequel tout finit toujours par s'arranger. C'est du moins ce que l'on se propose de dégager en examinant le contexte et la nature du voyage, la signification de l'arrière-plan mythologique du roman, et l'aboutissement de ce « road trip » en fonction de la pulsion de mort (ou du death drive). Ainsi, on mettra

\footnotetext{
${ }^{1}$ Voir entre autres à ce propos les compte-rendu du roman de Paul-André Proulx et de Kim Chabot.

${ }^{2} \mathrm{Au}$ sujet du « road novel », voir Romans de la route et voyages identitaires (2006).

${ }^{3}$ Guay-Poliquin ouvre son essai, Au-delà de la fin : Mémoire et survie du politique, en disant: « Survivant selon certains, anéanti selon d'autres, le projet moderne basé sur la foi envers le progrès et l'amélioration constante de la condition humaine semble s'être effondré sous les gravats de l'histoire » (9).
} 
en évidence les stratégies employées par ce roman pour subvertir l'idéal capitaliste sans tomber dans un discours nihiliste.

\section{L'inversion du projet occidental expansionniste : le cul-de-sac}

Doté d'un titre qui renvoie à la longueur du voyage du protagoniste et qui fait indirectement allusion au fil d'Ariane dans la mythologie grecque, Le fil des kilomètres joue sur l'anticipation, la liberté associée à la route, et l'idée que l'on peut surmonter les obstacles de la vie avant de déconstruire cette notion au tout début du roman. En effet, en ouvrant le roman, le lecteur fait face à une sorte de résumé de l'histoire à venir, présenté sous forme poétique, qui dévoile l'accident de voiture au dénouement de l'histoire et qui préfigure ce dont on est témoin au fur et à mesure que l'on avance dans le texte : l'effilochement du fil (du) conducteur et donc, du progrès. Bien que le défilement de la route devant le protagoniste, annoncé par le titre, soit d'abord associé à l'émancipation et à la filiation, il renvoie en réalité à une sorte de « unraveling » dans l'histoire et à la déconstruction du happy ending associé au rêve américain. Implicite, certes, cette interprétation du titre est confirmée par la description répétée de l'épuisement progressif du protagoniste au cours du voyage et par la conclusion qui rappelle que rien ne se passe jamais comme prévu et qu'il existe toujours des imprévus sur la route. Cette dernière, bien tracée au départ, est effectivement bouleversée à la fin du récit par l'accident, qui ramène le lecteur au début du roman où l'on met en scène une vision dystopique de l'ouest et donc, du monde occidental industrialisé.

Au début du récit principal qui commence à la suite de cinq courts chapitres, composés chacun de petits paragraphes décrivant le labyrinthe, puis l'arrivée d'un jeune mercenaire, le narrateur déclare : «Début de l'après-midi. Tout vient de s'arrêter. Plus de bruit. Il fait noir comme dans le ventre d'un moteur en panne » (15). Annonçant ici la panne d'électricité au centre de l'intrigue, l'incipit laisse entrevoir le caractère dystopique du point de départ du récit, une ville pétrolifère anonyme qui rappelle Fort McMurray, en Alberta. ${ }^{4}$ Plongée dans la noirceur, sous la pluie, la ville, qui fonctionne grâce à une raffinerie dont les flammes « trônent jour et nuit » (20) et projettent un halo orange, devient ce qu'elle est en réalité, c'est-à-dire un lieu infernal, peu « raffiné » et fort industriel, dépourvu de personnalité et de vie : "La ville s'étend avec des allures d'immense chantier en désordre. Tout est en construction. La population grimpe, mais elle n'est constituée que des travailleurs qui arrivent et repartent aussitôt leurs contrats achevés. ... quand le vent souffle, on entend le bourdonnement des camions, le claquement des pelles mécaniques et le rugissement des dinosaures de la raffinerie » (24). Associée paradoxalement ici à la préhistoire et au désordre alors qu'elle est censée incarner l'apogée du progrès industrialisé, la " cité » représente une sorte de dystopie capitaliste, dont les citoyens sont réifiés par le travail qui les occupe "vingt-quatre heures sur vingt-quatre » (27). Les gens, y compris le protagoniste qui travaille « $[\mathrm{d}] \mathrm{ix}$ heures par jour, sept jours par semaine » (25),

\footnotetext{
${ }^{4}$ Voir le premier chapitre de l'essai de Guay-Poliquin qui examine la question du genre utopique et dystopique dans le cadre de son étude de trois romans d'Antoine Volodine, de Marc Villemain et de Pavel Hak.
} 
constituent des espèces d'automatons dans un « royaume de la poussière » (25) dont l'horaire est destiné à maximiser les profits économiques : «Chacun son quart de travail. Quand on va se coucher, d'autres se lèvent et on se croise comme dans les rêves dont personne ne se souvient » (28).

Fort ironique, la situation à l'origine du récit où l'on décrit tout en fonction d'un monde animalesque laisse entendre que le monde occidental est cassé, que le rêve américain constitue en réalité un cauchemar où « [t] ous les jours finissent par se ressembler. Sans que rien ne change » (28). Dans le récit où les chantiers rappellent " une baleine échouée » (28), il n'y a pas de régénération; et la lumière, due au soleil qui ne se couche pas, est une source de peine et non pas un élément bienfaisant associé au progrès. ${ }^{5}$ Venu dans l'ouest avec une amie après avoir été mis à la porte par son père pour une raison inexpliquée (58), le protagoniste se trouve impuissant et inutile devant la panne d'électricité. Même en tant que mécanicien, il ne peut rien réparer. Il a besoin d'électricité, de ce courant vital, pour réparer les machines employées dans l'exploitation du pétrole. Ainsi, l'ouest dans le récit n'est pas le paradis annoncé dans le dicton célèbre «Go West, young man $»,{ }^{6}$ mais plutôt une sorte de non-lieu fragile qui cesse de fonctionner lorsque l'on coupe l'énergie qui l'alimente : « Tout est enfoui dans l'obscurité » (34).

Dans le texte, on est témoin de l'inversion de la conquête l'ouest dans la mesure où le protagoniste entreprend un voyage non dans cette direction, mais vers l'est du pays sans nom. Ayant été abandonné par l'amie avec qui il est arrivé dans la cité pétrolifère « neuf mois » $(30,65)$ avant la panne, le narrateur retourne au "berceau" en décidant de rentrer dans son village natal pour s'occuper de son père qui souffre d'une sorte d'Alzheimer « le cancer de la mémoire » (33), exacerbé par la panne d'électricité (31). En allant « vers [s]on village. Vers la maison. Vers [s]on père » (35) qui habite un " ancien village minier» (32), il entreprend un voyage régressif, un retour dans le temps pour essayer de réparer une autre situation dégénérative, et sans issue : "Pour racheter quelques erreurs, aussi. Et défier le passé » (36). Motivé par de nobles intentions et par la crise, le protagoniste va à l'encontre de la théorie du Manifest Destiny, dans le sens où il va dans la direction opposée des gens «qui fuient vers l'ouest du pays » (132). En effet, le narrateur entreprend l'opposé du voyage de Columbia, la personnification des États-Unis dans le tableau American Progress (1872) de John Glast qui pose un fil télégraphique, apportant la lumière à une région, sombre et peu développée. ${ }^{7}$

Dans le roman, le concept du Manifest Destiny, à l'origine de la fondation de cette ville pétrolifère dans l'ouest, est subverti. Au lieu de renvoyer à l'expansion territoriale naturelle et prédéterminée des Américains, le terme anticipe

\footnotetext{
${ }^{5}$ Le narrateur laisse entendre que les citoyens sont aveuglés par la lumière, l'artifice du progrès, lorsqu'il déclare : «Je scrute rarement le ciel. En plus, du village, on ne le voit pas vraiment. À cause des lumières de la raffinerie » (21).

${ }^{6}$ La phrase est attribuée à l'auteur et à l'éditeur du journal, Horace Greeley, qui favorisait l'expansion de l'ouest.

${ }^{7}$ Le terme est inventé par John L. Sullivan en 1845 et associé à l'expansionnisme américain. Pour une explication détaillée et complète du Manifest Destiny, voir Amy Greenberg dont l'étude affiche le tableau de Glast sur la couverture. Dans le tableau, le fil télégraphique ressemble au fil électrique.
} 
obliquement la nature cauchemardesque de la fin du voyage du protagoniste, préfigurée au début du roman et dans les chapitres mythologiques. Il renvoie à l'ironie du sort du protagoniste et souligne l'importance du hasard et de l'imprévisible dans la vie. Tracassé par le sentiment d'être talonné par son passé qu'il décrit comme ayant « une tête affreuse, des cornes et une gueule béante » (40), le protagoniste décrit son voyage en fonction du destin. Il est propulsé vers sa destination par l'appel (téléphonique) de son père, par un devoir filial. Il répète à diverses reprises qu'il veut revoir son père (37) et ne remet jamais en question le voyage qui le plonge dans un monde de plus en plus (post) apocalyptique et qui semble ainsi manquer justement de «sens ».

En allant vers l'est, le narrateur décrit la plaine comme une «mer» et entreprend un voyage mémoriel et macabre : il se remémore « la mort » (58) de sa mère dans un accident de voiture, la fermeture de la mine où travaillait son père, et fait face à une situation noire et anarchique où règne le chaos. ${ }^{8}$ En voyant un train se faire piller par des gens, il déclare : «On dirait presque des charognards se rassasiant d'une carcasse fraîche » (64). Avançant dans un monde de moins en moins civilisé qui rappelle paradoxalement «the Wild West» où les gens sont armés et il n'y a pas de règles, le narrateur entreprend un voyage conradien, aux cœurs des ténèbres où le familier devient l'opposé. ${ }^{9}$ Le lecteur constate une certaine étrangeté troublante quand il décrit certains endroits comme étant là où « le cœur du monde » a cessé de battre et lorsqu'il imagine la chaussée l'avalant « avec la patience d'un reptile qui digère sa proie » (132). Associée traditionnellement à la liberté et au rêve américain, la route incarne un serpent ici et renvoie ainsi à la nature traître et illusoire de ces objectifs. Loin de constituer un accès direct vers l'émancipation, elle rappelle un prédateur qui trompe ses victimes en leur faisant croire que sa forme rectilinéaire, facile à suivre en apparence, mène au bonheur.

Obsédé par la route et sa destination, le protagoniste, qui connaît toutes sortes de péripéties ou de « détours $»^{10}$ avant d'arriver à sa destination avec une jeune femme qu'il a laissée monter dans sa voiture et qui lui rappelle son ancienne amie, se trouve prisonnier dans une sorte de labyrinthe. Vers la fin du voyage, lorsqu'il arrive dans son pays natal, ${ }^{11}$ roulant de nuit tout en buvant un "liquide

\footnotetext{
${ }^{8}$ En rapportant les propos de son père au téléphone, le narrateur explique « des gens étaient venus chez lui. Qu'ils voulaient les clés de la voiture, la nourriture, l'argent. Ils sont entrés et ont gueulé très fort ... . Ils étaient quatre. Ils ont fouillé la maison en renversant tout » (31).

${ }^{9}$ Au coeur des ténèbres (1899), intitulé Heart of Darkness en anglais, raconte l'histoire du voyage de Charles Marlow qui remonte le cours d'un fleuve au cœur de l'Afrique noire. Embauché par une compagnie belge, il doit rétablir des liens commerciaux avec le directeur d'un comptoir au cœur de la jungle, Kurtz, très efficace collecteur d'ivoire mais dont on est sans nouvelles.

${ }^{10}$ Lors d'un arrêt à un hôtel avec la femme, le protagoniste se voit obligé de prendre à bord un homme armé qui finit par être « un complice de son ancien copain qui est devenu son amant. Après l'accident » (167). Cet homme raconte des histoires potentiellement mensongères qui remettent en question la nature absolue de la vérité.

${ }^{11}$ En parlant des nuages, il explique que c'est « comme si une immense main s'apprêtait à saisir le monde. Bientôt, il ne restera entre le ciel et la terre que l'espace d'une voiture » (175).
} 
clair", il rêve qu'il se rend chez son père ${ }^{12}$ et s'aperçoit qu'il somnole. Reprenant le contrôle de la voiture, il donne le volant à sa compagne qui écrase par accident le père du narrateur alors que celui-ci cherchait en pleine nuit l'endroit où sa femme avait trouvé la mort. Imprévisible sur le plan narratif et très ironique, l'accident, au cours duquel la voiture se retrouve sur le toit, "renverse" la fortune du protagoniste et laisse entendre qu'il y a des angles morts dans la vie. Retourné momentanément dans une position fœtale dans son véhicule en raison de la consommation d' « un liquide clair » (183), le protagoniste est trahi par son rêve. En effet, cet «alcool» (183) qui rappelle paradoxalement «l'eau de vie » et le liquide amniotique renvoie au flux caractérisant l'existence humaine et participe à l'accident dont l'avènement fait comprendre que l'on demeure, malgré les efforts pour rester sur le bon chemin, sujet à l'imprévisible, aux pulsions, et enfin de compte à la loi de Murphy selon laquelle « tout ce qui est susceptible de mal tourner tournera mal ».

\section{Le Minotaure et l'explication psychanalytique partielle}

S'étant endormi avant l'accident en buvant ce liquide clair qui rappelle celui que son père dépressif buvait suite à la mort de sa femme ${ }^{13}$ dans une voiture qu'il « venait tout juste de réparer » (59), le narrateur se réveille dans son village natal, les jambes cassées, découvre la tragédie et constate que les gens qui l'ont sauvé n'ont pas retrouvé sa compagne : «Quelle femme ? Il n'y avait aucune personne du côté passager » (197, je souligne). Mettant en doute l'existence réelle de cette femme, cette déclaration renvoie au caractère énigmatique de l'accident et laisse entendre que le narrateur, dans le contexte mythologique, n'est pas sorti du labyrinthe qu'est la vie. Il n'a pas réussi à sauver son père que les villageois ont laissé «filer » (197) dans la nature suite à ses réclamations pour aller retrouver « son épouse » (197) et a paradoxalement détruit le symbole d'ordre qu'il cherchait à sauver. Victime de la logique collective aux prises avec l'irrationalité du père, le narrateur rappelle une figure œdipienne, mais ne paraît pas excessivement rattaché à sa mère, décédée quand il avait 16 ans, et n'est pas explicitement associé à CEdipe dans le roman, mais plutôt à Thésée.

S'il constitue une version de Thésée comme le suggèrent les neuf chapitres « mythologiques » du roman, il arrive à tuer la bête qu'est son père et qui menace le village, mais semble en fin de compte avoir perdu le fil d'Ariane. Et, quoique cet événement par rapport au destin, il est clair que la mort du père n'est pas un acte de vengeance ou un acte inconscient commis par le protagoniste, mais un accident provoqué par sa compagne qui a voulu l'accompagner «jusqu'à [sa] destination » (174). Ainsi, l'explication mythique de l'événement ne fonctionne

\footnotetext{
${ }^{12}$ Voir « Kilomètre 4664 » (178). où le narrateur s'en dort brièvement en conduisant et fait un rêve où il arrive chez son père, avant de récupérer le contrôle de la voiture et de donner le volant à sa compagne.

${ }^{13}$ Après avoir évoqué l'histoire du décès accidentel de sa mère (Kilomètre 1023), le narrateur explique en parlant de son père qu' ' 'il se débattait avec ses propres ténèbres. Un peu plus tard, il avait recommencé à boire ce liquide clair qui lui arrachait la gorge et la voix » (60).
} 
que de manière superficielle. Quant à la jeune femme, que l'on ne peut qu'associer à Ariane, elle n'aide pas le protagoniste à sortir du labyrinthe, bien au contraire. Lui ayant offert le liquide clair au moment de prendre le volant (183), elle s'apparente à un pharmakon - un poison/remède -, qui plonge le narrateur non dans la lucidité, mais dans la noirceur : celle du sommeil et celle d'un scénario cauchemardesque. Sa disparition mystérieuse par la suite va aussi à l'encontre de ce qui se passe dans le mythe où c'est normalement Thésée qui abandonne Ariane endormie une fois arrivé à l'île Dia (divine) ou Naxos. Sorte d'énigme, la femme dont l'absence suite à l'accident reste inexpliquée, fait comprendre qu'il existe plusieurs conclusions par rapport au sort d'Ariane dans ce mythe. Dans une version du mythe, Ariane est sauvée et devient la compagne de Dionysos, alors qu'ailleurs elle se pend, péripétie évoquée par le narrateur lorsqu'il se rappelle son abandon par sa première compagne qui, après lui avoir fait l'amour dans son atelier mécanique, a tenté de se pendre à une chaîne : "Ses pieds se débattaient dans le vide. Ses poings étaient fermés. Ses yeux arqués ver le plafond. »(66). Ayant réussi à sauver celle-ci, qui ne veut «plus rien savoir. De cette ville, de cette vie, Et de moi surtout » (67), le protagoniste agit à l'opposé de Thésée.

Ainsi, l'explication de l'accident par la mythologie et la psychanalyse s'avère fallacieuse. Il existe, certes, des liens rattachant les deux histoires, mais l'on perd à la longue le fil d'Ariane : il s'effiloche, plongeant le lecteur dans le doute et le laissant avec toutes sortes de questions. En examinant de près les neuf chapitres portant sur le mythe de Thésée, là encore, rien n'est clair. Le mythe dans le roman ne suit pas la version traditionnelle, voire hollywoodienne, selon laquelle Thésée surmonte l'obstacle, sort du labyrinthe, et s'en va avec la femme dans les bras. Le neuvième chapitre du roman, nommé explicitement «Le fil d'Ariane » et qui suit la description cinématographique, par bribes, de l'accident, décrit une situation dans laquelle le jeune mercenaire semble s'être fait des illusions quant à la tuerie de la bête. Narré à la troisième personne, le passage qui fait penser à une sorte de fantasme intra-utérin par la mention du « fil rouge qui est à bout de souffle » (75) laisse entendre que le protagoniste ressemble à un nouveau né, sorti du labyrinthe, voire du ventre de la mère, ne serait-ce que pour se retrouver dans un autre labyrinthe - le monde. Autrement dit, on semble avoir affaire à la toute fin du passage à un nouveau-né, qui se voyait comme Thésée et qui défie la mort au moment de la naissance, mais qui se trouve une fois dehors dans une autre sorte de labyrinthe, le monde qui l'enveloppe, l'emmaillote : « Chaque fois qu'il relève la tête, il croit retrouver son chemin et être en mesure d'évaluer où se dissimule la sortie. Cela le rassure même si, en réalité, il ne fait que s'agiter dans un labyrinthe qui se resserre inévitablement sur lui » (188).

Ce qui est compréhensible dans cette description reste toutefois quelque peu ambigu. Ni le sens du passage précédent, ni celui de la sortie que voit le jeune mercenaire ne sont explicites. Le lecteur arrive à suivre le «passage » jusqu'à un certain point, mais le sens final et l'objectif de la description lui échappent. Les liens sémantiques n'ont plus de sens; le tout n'est pas clair, à part le fait qu'il n'y a pas de solution, de sortie réelle. Doté d'une conclusion assez pessimiste, le mythe revêt ici un caractère autoréflexif et laisse entendre que l'on se fait des illusions par 
rapport à la réalité ; on n'arrive pas à s'en sortir. Le monde n'est ni entièrement explicable, ni maîtrisable ; croire le contraire et se prendre pour un héros capable de défaire cette bête hybride et aléatoire relève justement du mythe, d'une construction imaginaire inventée pour rendre compte de l'inexplicable.

Réparti en neuf chapitres, ce composant mythologique du roman fait penser à la gestation, mais non à la naissance, à la victoire, et à la réussite, voire au succès. Au contraire, elle fait plutôt penser à une sorte de rupture et à un recommencement, idée évoquée lorsque l'on pense à la rupture du narrateur avec sa première compagne après justement neuf mois. Abandonné du jour au lendemain par celleci, le narrateur est plongé en allant vers l'est dans une autre aventure aussi, voire plus, complexe que la première. En effet, c'est que l'on voit dans le deuxième roman de Guay-Poliquin, Le poids de la neige (2016) qui raconte l'aventure d'un personnage qui se remet de fractures aux jambes au cours d'un hiver éternel dans un contexte apocalyptique suite à un accident de voiture ayant provoqué la mort de son père. Le lien avec Le fil des kilomètres est implicite, pas tout à fait clair, ${ }^{14}$ mais affecte la compréhension de l'histoire jusqu'à un certain point, comme les liens entre le protagoniste et le jeune mercenaire qui ressemble à Thésée.

\section{Éros et la pulsion de mort ou death drive}

Nourrie par un arrière-fond mythique, l'histoire au centre du Fil des kilomètres peut se comprendre en fonction des pulsions de vie et de mort, telles que définies par Freud. Dans le contexte du récit, le narrateur, qui rappelle une sorte de fils prodigue dérisoire, part vers l'est pour «rattraper le temps perdu », se réconcilier avec son père, et s'occuper de lui. Ses intentions sont nobles : il est motivé par l'amour, souhaite renouer avec celui qui l'a abandonné après avoir été abandonné par une femme, puis retrouve une sorte de compagne en route. En prenant la route, il recherche une certaine plénitude que l'on trouve paradoxalement selon Freud dans les deux pulsions opposées, dans le combat entre la pulsion de vie et la pulsion de mort.

D'abord, la pulsion de vie que Freud définit en fonction de l'autoconservation et la sexualité, la liaison, la compulsion de répétition dans son versant adaptatif et le principe de plaisir, elle peut s'employer pour décrire en partie le voyage du protagoniste. ${ }^{15}$ Confronté au début du récit à une panne d'électricité et à ce qui paraît être une situation sans issue, et donc, à une sorte de mort, il cherche à se redonner la vie, à renouer avec son père, et satisfait en cours de route à sa libido en plus de vivre une séries d'aventures excitantes, qui rappellent parfois des scènes tirées de films d'actions. ${ }^{16}$ Il recherche le plaisir et est travaillé par le désir, ce manque insatisfaisable qui est "increvable » (29) comme le «moteur» de sa voiture dont « la mécanique est simple. Quand ça se brise, ça se répare, ça se

\footnotetext{
${ }^{14}$ Voir à ce propos le blogue de Gabrielle Vallières.

${ }^{15}$ Voir « Au-delà du principe de plaisir » où Freud aborde cette pulsion et celle de la mort.

16 Voir la note No. 10 et les chapitres entre « Kilomètre 3921 » (146) et « Kilomètres 4232 » (173)

où le narrateur vit des péripéties qui rappellent en partie celles que l'on voit dans des westerns.
} 
rafistole » (29). Le narrateur, qui n'abandonne jamais sa voiture, entreprend un voyage vers la mort, un death drive, étant donné l'aboutissement fatal du voyage.

Ayant entrepris le voyage pour revoir son père - incarnation de la loi et de la punition - qui lui a dit de ne plus jamais revenir, le protagoniste remonte dans le passé et s'investit contre toute attente dans un exploit qui risque de mal finir. En effet, le coup de fil du père à l'origine du voyage signale à quel point le protagoniste est resté attaché de façon ombilicale à ce passé qu'il imagine comme une bête qui le pourchasse et qu'il évoque dans un geste répétitif, compulsif. ${ }^{17}$ Cette répétition d'un traumatisme, du refoulé, se trouve au centre de la pulsion de mort que Freud définit en fonction d'un désir de ramener le vivant à un état antérieur anorganique. ${ }^{18}$ Dans l'histoire, le protagoniste est à la recherche d'une certaine plénitude (associée au foyer familial) et se protège constamment, tout en buvant de la bière, liquide qui nuit à sa lucidité.

Son plaisir participe de sa destruction, de même que la pulsion de mort est liée au surmoi, ce que l'on constate à la fin du roman lorsqu'il semble boire ce que son père buvait jadis pour refouler la douleur associée au décès de sa femme. S'étant laissé aller à un geste destructeur et au sommeil, cet état confortant qui rappelle l'expérience intra-utérine soulevée dans le neuvième chapitre, le narrateur régresse momentanément et reproduit autrement le traumatisme de l'accident ayant tué sa mère. Son voyage ne relève ainsi que d'une "sorte » de death drive, puisqu'il n'est pas le conducteur de la voiture et ne constitue pas en fin de compte la cible de la pulsion de mort ; il survit à l'accident. En tuant par accident son père, il déconstruit la loi, et laisse entendre que rien n'est absolu, comme le signale Freud en parlant du « combat » éternel entre ces deux pulsions. ${ }^{19}$ L'accident et la survie $\mathrm{du}$ protagoniste subvertissent les explications de la situation et font revenir le lecteur au début du roman, mais de façon différente.

Son voyage met fin à la souffrance de son père que les villageois enterrent rapidement, mais l'accident laisse le narrateur avec une autre sorte de traumatisme, et fait de lui, le remplaçant de son père. Le dérapage de la voiture dans le même virage où sa mère a perdu contrôle de sa propre voiture, incarne un glissement symbolique au sens derridien du terme et montre que la route, comme la vie, contient toutes sortes de virages et d'imprévus. Le chemin devant soi, qu'il s'agisse de projets futurs ou de la tentative de réparer le passé, n'est pas toujours droit. Dans le cas de la mère du protagoniste, " un grand coup de volant» (60) inexpliqué provoque son accident et c'est aussi ce qui semble avoir provoqué le sien. L'amie du narrateur donne un « coup de volant » (186) à la voiture pour éviter le père dont les « deux yeux » (186), tels ceux d'une bête, luisaient au milieu de la route dans la

\footnotetext{
${ }^{17}$ « Je ne veux plus n'être pour lui qu'un nom à l'autre bout du monde. Il a besoin de moi, je le sais. ... Et moi, j'ai besoin de lui. Pour être sûr de lui ressembler le moins possible » (157).

${ }^{18}$ « Le but de l'Éros est d'établir des unités toujours plus grandes afin de les conserver : en un mot, un but de liaison. Le but de l'autre instinct, au contraire, est de briser tous les rapports, donc de détruire toute chose. Il nous est permis de penser de l'instinct de destruction que son but final est de ramener ce qui vit à l'état inorganique et c'est pourquoi nous l'appelons instinct de mort » (Abrégé de psychanalyse, 8 ).

${ }^{19}$ Voir à ce propos, Le malaise dans la culture (1929).
} 
noirceur à l'extérieur du village. En effet, comme un animal qui surgit sur la route, le père surprend la conductrice qui à son tour aveugle sa victime par accident. Métaphorique, la scène représente la nature paradoxale de la vie où, si la lumière aide à voir devant soi et prévient les accidents, elle peut y contribuer aussi.

L'ironie de l'accident porte sur la question de l'aveuglement et le paradoxe pascalien selon lequel « qui veut faire l'ange fait la bête » (Frag 572). L'accident souligne l'importance du «ça», de l'impulsivité, de la «bêtise» au sein du «savoir», cette lumière, associée au pouvoir, à l'agentivité, et déconstruit les illusions que l'on se fait par rapport à la vie, ce phénomène " bête », pour ne pas dire monstrueux, défini par le hasard et l'imprévisible. À ce propos, il me semble possible de résumer l'accident et enfin l'ironie caractérisant le roman en faisant référence à la gravure célèbre de Francisco Goya El sueño de la razon produce monstruos (c.1799). Le rêve de la Raison, soit la croyance que l'on a raison et que l'on peut associer à la conductrice dans le récit, de même que le sommeil de la Raison - ici le manque de vigilance du protagoniste qui s'endort - produisent tous deux des monstres, c'est-à-dire des situations affreuses.

\section{Conclusion : la sur-vie}

Doté ainsi d'un message qui rappelle le dilemme cornélien, Le fil des kilomètres met en scène la déconstruction du Sens, de la direction, des idéologies, et des solutions toutes faites que l'on adopte pour comprendre le monde et y faire face. Fataliste dans le sens où il laisse entendre que l'on n'arrive jamais à sortir tout à fait du labyrinthe, à maîtriser la nature aléatoire de la vie et que la route de l'enfer est pavée de bonnes intentions, le roman n'est pas cependant nihiliste. Il s'agit dans ce récit, à la fois simple et complexe, d'une mise en garde contre les illusions que l'on se fait sur soi-même, et vis-à-vis de la croyance qui voudrait que la vie peut changer. L'histoire montre que la vie est cyclique, mais que cette dernière réalité, en faisant penser à l'éternel retour et à l'enfer, est aussi porteuse d'espoir comme dans «le mythe" de Sisyphe. Cependant, loin d'afficher une position existentialiste, le roman se termine tout compte fait sur une note positive et fait comprendre qu'il faut, malgré tout, garder la foi : le protagoniste, immobile dans un premier temps, connaît la mobilité avant d'être immobilisé, mais reste en vie et dans le dénouement se trouve sur la voie du rétablissement.

Ressuscité après « trois jours » (195) tel Jésus, le protagoniste n'est pas le messie : il a tué son père et a les jambes cassées à la fin de l'histoire. ${ }^{20}$ Il est cependant sauvé et signale par sa survie que l'humanité, malgré sa cécité, n'est pas totalement à mépriser et qu'il existe pour ainsi dire une lumière au bout du tunnel, de l'espoir au fond de la boîte de Pandore qu'est la vie. À la fin du roman, le lecteur est effectivement témoin d'une sorte de renaissance de l'homme et d'un enlightenment qui valorise la survie et la compulsion d'aller de l'avant tout en faisant comprendre qu'il faut tenir compte des erreurs passées pour bien avancer. ${ }^{21}$

\footnotetext{
${ }^{20}$ Voir à ce propos Jean 19:31-37 qui confirme Exode 12:46, Nombres 9:12, et Éphésiens 5:27.

${ }^{21}$ Cette conclusion se rapporte en grande partie à la thèse de l'essai de Guay-Poliquin : «Les protagonistes de notre corpus d'études tentent de 'réveiller les morts et rassembler ce qui a été
} 
À ce propos, les dernières phrases font allusion à l'importance du dialogue et au besoin de rester conscient de la nature paradoxale de la condition humaine, ce que l'on constate quand les villageois demandent le nom du protagoniste : "On n'a presque plus de passé. Mais il ne faut pas avoir peur. Ici, à défaut d'avoir des souvenirs précis, on arrive toujours à survivre. C'est ce que l'on a toujours fait d'ailleurs. Bon, allez, votre nom ? Dites-moi votre nom ? » (198). Représentatif de l'ironie que l'on retrouve tout au long du roman, la clausule joue sur l'imperfection des êtres humains qui oublient, qui répètent les erreurs du passé en allant de l'avant, mais semble également valoriser la résilience, la curiosité, et la bonté fondamentale des gens face à l'adversité du monde.

Aidé par des pairs inconnus mais qui le connaissent sans pourtant le reconnaître, le protagoniste n'est peut-être pas immédiatement prêt à affronter le monde, mais le sera dans l'avenir. C'est en effet ce que Guay-Poliquin nous montre dans son deuxième roman qui, en restant fidèle à la déconstruction du sens dont on est témoin ici, est et n'est pas la suite du premier.

démembré' de manière à assurer l'héritage de l'histoire passé et, par conséquent, la possibilité d'un avenir au-delà de ces multiples fins annoncées » (13). 


\section{Bibliographie}

Chabot, Kim. « Critique du livre Le fil des kilomètres de Christian GuayPoliquin. » Impact Campus, $25 \quad$ novembre http://impactcampus.ca/arts-et-culture/critique-du-livre-le-fil-des2013, kilometres-de-christian-guay-poliquin/.

Conrad, Joseph. Au cœur des ténèbres. Trad. Catherine Pappo-Musard. Paris : Livre de poche, 2012.

Freud, Sigmund. « Au-delà du principe de plaisir » (1920). Essais de psychanalyse. Traduit par S. Jankélévitch. Paris : Éditions Payot, 1968, pp. 7-82.

---. Le malaise dans la culture. 1929. Traduit par P. Cotet, R. Lainé et J. StuteCadiot. Paris : PUF, 1994.

---. Abrégé de psychanalyse. 1938. Traduit par Anne Berman. Paris : PUF, 1998.

Greenberg, Amy. Manifest Destiny and Territorial Expansion: A Brief History with Documents. Boston/New York : Bedford/St. Martins, 2017.

Guay-Poliquin, Christian. Au-delà de la fin. Mémoire et survie du politique. Québec : Presses de 1’Université du Québec, « Coll. Mnémosyne. » 2014.

---. Le fil des kilomètres. Montréal : Bibliothèque québécoise, 2016.

---. Le poids de la neige. Montréal : La Peuplade, 2016.

Morency, Jean, J. den Toonder et J. Lintvelt. Romans de la route et voyages identitaires. Montréal: Nota bene, 2006.

Pascal, Blaise. Pensées. 1670. Paris : Gallimard, 1977.

Proulx, Paul-André. "Le fil des kilomètres » Littérature québécoise. http://www.litterature-quebecoise.com/oeuvres/lefildekilometres.html

Vallières, Gabrielle. « Christian Guay-Poliquin - Une œuvre duelle. » Portraits. https://www.blogues.cstip.ulaval.ca/portraits/christian-guay-poliquin 